\title{
Nonclassical properties of non-Gaussian two-mode quantum vortex state
}

\author{
Vikram Singh $^{1 *}$, Devendra Kumar Mishra ${ }^{2}$ \\ ${ }^{1}$ Physics Department, Nehru Gram Bharati (Deemed to be University), Kotwa-Jamunipur Dubawal, Allahabad, U. P., India \\ ${ }^{2}$ Physics Department, Institute of Science, Banaras Hindu University, Varanasi-221005, India \\ *Corresponding Author: vikramsinghphy@gmail.com, Tel.: +919450254530
}

Available online at: www.isroset.org

Accepted: 06/Aug/2018, Online 31/Aug/2018

\begin{abstract}
Nonclassicality of optical fields are the key resources in quantum information processing. Recently, Agarwal [New J. Phys., 13, 073008(2011)] proposed a method for engineering of a two-mode vortex state, a non-Gaussian state, by subtracting a photon from a two-mode squeezed state produced by down-converter from idler mode via a beam splitter with low reflectivity and detection of one photon by the avalanche photo diode (APD), or even better by a single-photon detector. We study the nonclassicalities of this state in terms of squeezing, antibunching, photon number correlation and distribution of photon number. In addition, we study higher-order nonclassical properties, viz., sum squeezing. We find that the non-Gaussian state shows strong nonclassical effects.
\end{abstract}

Keywords-Nonclassicality, Non-Gaussian states, qantum vortex states, photon number correlation, two-mode squeezed states, sum squeezing

\section{INTRODUCTION}

The discovery of photoelectric effect in 1905 by Einstein may be regarded as the birth of quantum optics but HanburyBrown and Twiss experiment, in the 1950s, on intensity correlations in stellar interferometry established the quantum optics as a separate subject. Discovery of new magical source of light, called the laser, motivated theoreticians to establish a general framework for the description of states of radiation. Sudarshan and Glauber, independently, introduced phase space distributions and formulated that the averages of normal-ordered products of field operators to be computed as integrals of the corresponding c-number functions. This formal correspondence between the quantum-mechanical and classical statistical descriptions of optical coherence functions is the essence of the optical equivalence theorem.

Observation of squeezing, antibunching, and the associated (but inequivalent) sub-Poissonian photon statistics laid the foundation of the study of "non-classical light", i. e, light with properties that essentially results from the quantum nature of light and cannot be described classically. These nonclassical light features are the key resources in quantum information processing [1].

Quantized single-mode electric field of frequency $\omega$ can be written as $\hat{E}(t)=E_{0}\left(\hat{a}^{-i \omega t}+\hat{a}^{\dagger} e^{i \omega t}\right)$, where $E_{0}$ is a constant and $\hat{\mathrm{a}}$ is annihilation operator of the radiation. Defining two Hermitian operators $\hat{X}_{1}$ and $\hat{X}_{2}$ by
$\hat{\mathrm{X}}_{1}=\frac{1}{2}\left(\hat{\mathrm{a}}+\hat{\mathrm{a}}^{\dagger}\right)$ and $\hat{\mathrm{X}}_{2}=\frac{1}{2 \mathrm{i}}\left(\hat{\mathrm{a}}-\hat{\mathrm{a}}^{\dagger}\right)$, with $\left[\hat{\mathrm{X}}_{1}, \hat{\mathrm{X}}_{2}\right]=\frac{\mathrm{i}}{2}$, we can write $\hat{E}(t)=E_{0}\left(\hat{X}_{1} \cos \omega t+\hat{X}_{2} \sin \omega t\right)$. According to the Heisenberg uncertainty relation we must have $\left\langle\left(\Delta \hat{\mathrm{X}}_{1}\right)^{2}\right\rangle\left\langle\left(\Delta \hat{\mathrm{X}}_{2}\right)^{2}\right\rangle \geq \frac{1}{16}$, where $\left\langle\left(\Delta \hat{\mathrm{X}}_{\mathrm{i}}\right)^{2}\right\rangle=\left\langle\hat{\mathrm{X}}_{\mathrm{i}}{ }^{2}\right\rangle-$ $\left\langle\hat{\mathrm{X}}_{\mathrm{i}}\right\rangle^{2},(\mathrm{i}=1,2)$. For an optical field in a coherent state, $|\alpha\rangle$, we have $\left\langle\left(\Delta \hat{\mathrm{X}}_{1}\right)^{2}\right\rangle=\left\langle\left(\Delta \hat{\mathrm{X}}_{2}\right)^{2}\right\rangle=\frac{1}{4}$, i. e., coherent state is a minimum uncertainty state. But, squeezed states have fluctuation below the minimum quantum fluctuations for one component of the radiation field, $\left\langle\left(\Delta \hat{\mathrm{X}}_{\mathrm{i}}\right)^{2}\right\rangle<\frac{1}{4}$, (i=1 or 2$)$.

Correlation function $\mathrm{G}^{(2)}$, measure of the correlation of light intensities at two space-time points, of the field in the state $|\psi\rangle$ is defined by

$$
\mathrm{G}^{(2)}(\mathrm{t}, \mathrm{t}+\tau)=\left\langle\psi\left|\hat{\mathrm{E}}^{-}(\mathrm{t}) \hat{\mathrm{E}}^{-}(\mathrm{t}+\tau) \hat{\mathrm{E}}^{+}(\mathrm{t}) \hat{\mathrm{E}}^{+}(\mathrm{t}+\tau)\right| \psi\right\rangle
$$

and the normalized correlation function, measure of conditional probability of detecting a photon at time $t+\tau$, given that a photon was detected at time $t$ is

$$
\mathrm{g}^{(2)}(\mathrm{t}, \mathrm{t}+\tau)=\frac{\left\langle\psi\left|\hat{\mathrm{E}}^{-}(\mathrm{t}) \hat{\mathrm{E}}^{-}(\mathrm{t}+\tau) \hat{\mathrm{E}}^{+}(\mathrm{t}) \hat{\mathrm{E}}^{+}(\mathrm{t}+\tau)\right| \psi\right\rangle}{\left\langle\psi\left|\hat{\mathrm{E}}^{-}(\mathrm{t}) \mathrm{E}^{+}(\mathrm{t})\right| \psi\right\rangle^{2}} .
$$


Correlation function of the field at the same time point is defined by

$$
\mathrm{g}^{(2)}(\mathrm{t}, \mathrm{t})=\mathrm{g}^{(2)}(0)=\left\langle\psi\left|\hat{\mathrm{a}}^{\dagger} \hat{\mathrm{a}}^{\dagger} \hat{a} \mathrm{a}\right| \psi\right\rangle /\left\langle\psi\left|\hat{\mathrm{a}}^{\dagger} \hat{\mathrm{a}}\right| \psi\right\rangle^{2} .
$$

Mandel Q-parameter is defined as,

$$
\mathrm{Q}=\left[\left\langle\psi\left|(\Delta \hat{\mathrm{N}})^{2}\right| \psi\right\rangle-\overline{\mathrm{n}}\right] / \overline{\mathrm{n}}=\overline{\mathrm{n}}\left[\mathrm{g}^{(2)}(0)-1\right] \text {. }
$$

The photon statistics is Poissonian if $\mathrm{Q}=0$, superPoissonian if $\mathrm{Q}>0$, and the sub-Poissonian if $0>\mathrm{Q} \geq-1$. For example, a number state $|\mathrm{n}\rangle$ exhibits sub-Poissonian photon statistics: $\left\langle(\Delta \hat{\mathrm{N}})^{2}\right\rangle=0,\langle\hat{\mathrm{N}}\rangle=\mathrm{n}$ and hence $\mathrm{Q}=-1$. Obviously, $\mathrm{Q}=0$ for a coherent state.

Density operator, $\hat{\rho}$, of any quantum state of singlemode radiation field can be written as, $\hat{\rho}=\int P(\alpha)|\alpha\rangle\langle\alpha| d^{2} \alpha$, where $P(\alpha)$ is the Sudarshan-Glauber diagonal coherent state P-representation termed as "P-function". A quantum state is said to be non-classical if the P-function "is less well behaved than a probability density", e.g., takes on negative values or becomes more singular than a delta function. For the normal ordering (annihilation operators to the right, creation operators to the left), one has, for a single-mode field:

$$
\left\langle\left(\hat{a}^{\dagger}\right)^{\mathrm{m}} \hat{\mathrm{a}}^{\mathrm{n}}\right\rangle=\operatorname{Tr}\left[\hat{\rho}\left(\hat{\mathrm{a}}^{\dagger}\right)^{\mathrm{m}} \hat{\mathrm{a}}^{\mathrm{n}}\right]=\int\left(\alpha^{*}\right)^{\mathrm{m}} \alpha^{\mathrm{n}} \mathrm{P}(\alpha) \mathrm{d}^{2} \alpha .
$$

For a coherent state, $\hat{\rho}=\left|\alpha_{0}\right\rangle\left\langle\alpha_{0}\right| \quad, \quad$ so that $\operatorname{Tr}\left[\hat{\rho}\left(\hat{\mathrm{a}}^{\dagger}\right)^{\mathrm{m}} \hat{\mathrm{a}}^{\mathrm{n}}\right]=\left(\alpha_{0}^{*}\right)^{\mathrm{m}} \alpha_{0}^{\mathrm{n}}$, which corresponds to $\mathrm{P}(\alpha)=\delta^{(2)}\left(\alpha-\alpha_{0}\right)$ in the above integral. Thus, the Pfunction of a coherent state may be taken as a delta function. In this sense, the coherent states lie "on the border" of the set of "classical" states because delta function is the most singular distribution admissible in the classical theory.

Now, we can write

$$
\begin{aligned}
\left\langle\left(\Delta \hat{\mathrm{X}}_{1}\right)^{2}\right\rangle= & \frac{1}{4}\left\langle\left(\hat{\mathrm{a}}+\hat{\mathrm{a}}^{\dagger}\right)^{2}-\left\langle\left(\hat{\mathrm{a}}+\hat{\mathrm{a}}^{\dagger}\right)\right\rangle^{2}\right\rangle=\frac{1}{4}\left[\left\langle\hat{\mathrm{a}}^{2}+\hat{\mathrm{a}}^{\dagger 2}+2 \hat{\mathrm{a}}^{\dagger} \hat{\mathrm{a}}+1\right\rangle\right. \\
& \left.-\left\langle\left(\hat{\mathrm{a}}+\hat{\mathrm{a}}^{\dagger}\right)\right\rangle^{2}\right]
\end{aligned}
$$

i. e.,

$$
\left\langle\left(\Delta \hat{\mathrm{X}}_{1}\right)^{2}\right\rangle=\frac{1}{4}\left\{1+\int \mathrm{d}^{2} \alpha \mathrm{P}(\alpha)\left[\left(\alpha+\alpha^{*}\right)^{2}-\left\langle\alpha+\alpha^{*}\right\rangle^{2}\right]\right\}
$$

and then squeezing can occur $\left\langle\left(\Delta \hat{\mathrm{X}}_{1}\right)^{2}\right\rangle<\frac{1}{4}$, only if $\mathrm{P}(\alpha)$ is not positive definite and, therefore, squeezed states are nonclassical in nature.

Again, if the state of the radiation field is described by the P-function, we can write

$$
\left.\mathrm{g}^{(2)}(0)=1+\left(1 /\left\langle|\alpha|^{2}\right\rangle^{2}\right) \int \mathrm{d} \alpha \mathrm{P}(\alpha)\left(|\alpha|^{2}-\left\langle|\alpha|^{2}\right\rangle\right)^{2}\right),
$$

and this shows that, for classical fields, with $P(\alpha) \geq 0$, one should have $g^{(2)}(0) \geq 0$. Therefore, the property $g^{(2)}(0)<1$, i.e., $\left\langle(\Delta \hat{\mathrm{N}})^{2}\right\rangle<\overline{\mathrm{n}}$ is the characteristic of nonclassical light which is called sub-Poissonian photon statistics. It is important to note that the condition for sub-Poissonian photon statistics, $\mathrm{g}^{(2)}(0)<1$, implies the occurrence of photon antibunching $\mathrm{g}^{(2)}(\mathrm{t}, \mathrm{t}+\tau)>\mathrm{g}^{(2)}(0)$ but the converse is not true.

In the present paper, Section I contains the introduction of nonclassicalities of optical fields. Section II contains the related work of the two-mode quantum vortex state. Section III contains the different nonclassical properties of optical fields to be exhibited by the two-mode quantum vortex state. Section IV contains the results and their discussions, and Section V contains the conclusions of the research work and reflects future directions.

\section{RELATED WORK}

Two-mode squeezed vacuum state is defined by

$|\xi\rangle=\exp \left(\xi \hat{a}^{\dagger} b^{\dagger}-\xi^{*} \hat{a} b\right)|0,0\rangle, \xi=\mathrm{re}^{\mathrm{i} \varphi}$,

where $\xi$ is a complex parameter, a and b represent two modes of the field with commutation relations $\left[\hat{\mathrm{a}}, \hat{\mathrm{a}}^{\dagger}\right]=\left[\hat{\mathrm{b}}, \hat{\mathrm{b}}^{\dagger}\right]=1,\left[\hat{\mathrm{a}}, \hat{\mathrm{b}}^{\dagger}\right]=0$, etc. The most fundamental process by which we study the interaction of matter with light is the addition or subtraction of a single photon from the radiation field. The ability to achieve this level of control in experiments has been used to produce novel non-classical states of light by the well-known process of "degaussification" and in a direct demonstration of the commutation relation between the annihilation and creation operators. Agarwal [2] proposed a novel method for engineering of a two-mode vortex state by subtracting [3] a photon from a two-mode squeezed state produced by down converter from idler mode $b$ via a beam splitter with low reflectivity and detection of one photon by the avalanche photo diode (APD), or even better by a single-photon detector. The beam splitter has reflectivity of the order of a few per cent so that the probability of finding two photons is of the order of $10^{-4}$. Several successful experiments have been done for the subtraction procedure upto nearly $100 \%$ accuracy. The resulting two-mode vortex state of the output field is

$$
|\xi\rangle^{(\mathrm{s})}=\mathrm{Nb} \exp \left(\xi \hat{a}^{\dagger} \hat{b}^{\dagger}-\xi^{*} \hat{a} \hat{b}\right)|0,0\rangle=\mathrm{e}^{\mathrm{i} \varphi} \hat{\mathrm{a}}^{\dagger}|\xi\rangle /(\cosh \mathrm{r}),(10)
$$

where, $N=\left\langle\xi\left|\hat{b}^{\dagger} \hat{b}\right| \xi\right\rangle^{-1 / 2}=1 / \sinh r$. Its Wigner function is [2] 
$\mathrm{W}^{(\mathrm{s})}(\alpha, \beta)=\frac{4}{\pi^{2}}\left(4|\tilde{\alpha}|^{2}-1\right) \exp \left[-2\left(|\tilde{\alpha}|^{2}+|\tilde{\beta}|^{2}\right)\right]$,

where $\tilde{\alpha}$ and $\tilde{\beta}$ are given by $\tilde{\alpha}=\alpha \cosh r-\beta^{*} \sinh r \mathrm{e}^{\mathrm{i} \varphi}, \tilde{\beta}^{*}=-\alpha \sinh r \mathrm{e}^{\mathrm{i} \varphi}+\beta^{*} \cosh \mathrm{r}$.

\section{NONCLASSICAL PROPERTIES OF TWO- MODE QUANTUM VORTEX STATE}

In discrete variables, the nonclassicality can be characterised in terms of coherence and entanglement. The difficulty of creating a quantum superposition of states in some designated classical basis (such as energy eigenstates) is termed as the coherence, whereas the entanglement characterises the difficulty of expressing the state of combined system as the product of the states of two individual systems. Whereas, in continuous variables, coherent states of light are the most classical pure states and because of which it is relatively easy synthesise these states. Coherent states are not mutually orthogonal, and represent a superposition of different energies. A state is termed as a nonclassical state when it is not a probabilistic mixture of coherent states. For Gaussian states, the calculations and analysis of nonclassicalities are relatively easy since the Gaussian states can defined in terms of the first and second moment. But, this is not so easy for the case of non-Gaussian state, for example, the two-mode quantum vortex state in the present paper. There are a number of nonclassical properties of optical fields. But, here we will focus to investigate the squeezing, antibunching, photon number correlation and distribution of photon number and the sum squeezing, as an example of the higher-order squeezing, of the two-mode quantum vortex state.

\section{A. Squeezing Properties:}

Because of the correlations between the modes, the squeezing of quantum fluctuations is not in the individual modes but rather in a superposition of the two-modes. We define the superposition quadrature operators as follows:

$\hat{\mathrm{X}}_{1}^{\mathrm{ab}}=\frac{1}{2^{3 / 2}}\left(\hat{\mathrm{a}}+\hat{\mathrm{a}}^{\dagger}+\hat{\mathrm{b}}+\hat{\mathrm{b}}^{\dagger}\right)=\frac{1}{\sqrt{2}}\left(\hat{\mathrm{X}}_{1}^{\mathrm{a}}+\hat{\mathrm{X}}_{1}^{\mathrm{b}}\right)$,

$\hat{\mathrm{X}}_{2}^{\mathrm{ab}}=\frac{1}{2^{3 / 2} \mathrm{i}}\left(\hat{\mathrm{a}}-\hat{\mathrm{a}}^{\dagger}+\hat{\mathrm{b}}-\hat{\mathrm{b}}^{\dagger}\right)=\frac{1}{\sqrt{2}}\left(\hat{\mathrm{X}}_{2}^{\mathrm{a}}+\hat{\mathrm{X}}_{2}^{\mathrm{b}}\right)$.

The operators satisfy the same commutation relations, $\left[\hat{\mathrm{X}}_{1}^{\mathrm{ab}}, \hat{\mathrm{X}}_{2}^{\mathrm{ab}}\right]=\mathrm{i} / 2$, and thus squeezing exists in the superposition quadratures if the conditions $\left\langle\left(\Delta \hat{\mathrm{X}}_{1}^{\mathrm{ab}}\right)^{2}\right\rangle<1 / 4$, or $\quad\left\langle\left(\Delta \hat{\mathrm{X}}_{2}^{\mathrm{ab}}\right)^{2}\right\rangle<1 / 4 \quad$ is $\quad$ satisfied. Here, $\left\langle\left(\Delta \hat{\mathrm{X}}_{\mathrm{i}}^{\mathrm{ab}}\right)^{2}\right\rangle \equiv\left\langle\left(\hat{\mathrm{X}}_{\mathrm{i}}^{\mathrm{ab}}\right)^{2}\right\rangle-\left\langle\hat{\mathrm{X}}_{\mathrm{i}}^{\mathrm{ab}}\right\rangle^{2}$

\section{B. Photon number correlation and distribution of photon number}

In order to quantify the quantum correlation between the two modes, we must examine operators acting on both systems. Here we take combination photon number operators $\hat{\mathbf{M}}_{ \pm}=\hat{\mathrm{n}}_{\mathrm{A}} \pm \hat{\mathrm{n}}_{\mathrm{B}}$ and examine the variances

$$
\left\langle\left(\Delta \hat{\mathrm{M}}_{ \pm}\right)^{2}\right\rangle=\left\langle\left(\Delta \hat{\mathrm{n}}_{\mathrm{A}}\right)^{2}\right\rangle+\left\langle\left(\Delta \hat{\mathrm{n}}_{\mathrm{B}}\right)^{2}\right\rangle \pm 2 \operatorname{Cov}\left(\hat{\mathrm{n}}_{\mathrm{A}}, \hat{\mathrm{n}}_{\mathrm{B}}\right)
$$

where, the covariance of the photon numbers is defined as

$\operatorname{Cov}\left(\hat{\mathrm{n}}_{\mathrm{A}}, \hat{\mathrm{n}}_{\mathrm{B}}\right) \equiv\left\langle\hat{\mathrm{n}}_{\mathrm{A}} \hat{\mathrm{n}}_{\mathrm{B}}\right\rangle-\left\langle\hat{\mathrm{n}}_{\mathrm{A}}\right\rangle\left\langle\hat{\mathrm{n}}_{\mathrm{B}}\right\rangle$.

According to classical probability theory, the Covariance of any two random variables, $\mathrm{X}$ and $\mathrm{Y}$, is

$\operatorname{Cov}(\mathrm{X}, \mathrm{Y}) \equiv\langle\mathrm{XY}\rangle-\langle\mathrm{X}\rangle\langle\mathrm{Y}\rangle$.

So, Covariance of any two observables $\hat{X}$ and $\hat{Y}$ is

$\operatorname{Cov}(\hat{X}, \hat{Y}) \equiv\langle\hat{X} \hat{Y}\rangle-\langle\hat{X}\rangle\langle\hat{Y}\rangle$.

Again from classical probability theory given two random variables, we define the linear correlation function

$J(X, Y)=\operatorname{Cov}(X, Y) /\left\langle(\Delta X)^{2}\right\rangle^{1 / 2}\left\langle(\Delta Y)^{2}\right\rangle^{1 / 2} \in[0,1]$.

Then, the linear correlation coefficient is

$\mathrm{J}\left(\hat{\mathrm{n}}_{\mathrm{A}}, \hat{\mathrm{n}}_{\mathrm{B}}\right)=\operatorname{Cov}\left(\hat{\mathrm{n}}_{\mathrm{A}}, \hat{\mathrm{n}}_{\mathrm{B}}\right) /\left\langle\left(\Delta \hat{\mathrm{n}}_{\mathrm{A}}\right)^{2}\right\rangle^{1 / 2}\left\langle\left(\Delta \hat{\mathrm{n}}_{\mathrm{A}}\right)^{2}\right\rangle^{1 / 2}$

When $\mathrm{J}\left(\hat{\mathrm{n}}_{\mathrm{A}}, \hat{\mathrm{n}}_{\mathrm{B}}\right)=1$, the maximal value indicating strong inter mode correlations. The joint probability of finding $n_{1}$ photons in mode $A$ and $n_{2}$ photons in mode $B, P_{n_{1} n_{2}}$ :

$\mathrm{P}_{\mathrm{n}_{1} \mathrm{n}_{2}}=\left|\left\langle\mathrm{n}_{1}, \mathrm{n}_{2} \mid \xi\right\rangle^{(\mathrm{s})}\right|^{2}$.

\section{Antibunching effect:}

The criterion for existence of antibunching in twomode radiation is given by [4]

$R_{a b}=\left(\left\langle\hat{a}^{\dagger} \hat{a}^{2}\right\rangle+\left\langle\hat{b}^{\dagger} \hat{b}^{2}\right\rangle\right) / 2\left\langle\hat{a}^{\dagger} \hat{a} \hat{b}^{\dagger} \hat{b}\right\rangle-1<0$.

\section{Sum Squeezing:}

Defining the sum quadrature operator for the two modes as

$\hat{\mathrm{X}}_{\phi}=\frac{1}{2}\left(\mathrm{e}^{\mathrm{i} \phi} \hat{\mathrm{a}} \hat{\mathrm{b}}+\mathrm{e}^{-\mathrm{i} \phi} \hat{\mathrm{a}}^{\dagger} \hat{\mathrm{b}}^{\dagger}\right)$,

the sum squeezing [5] condition becomes

$\Delta \hat{\mathrm{X}}_{\phi}^{2}<\frac{1}{2}\left|\left[\hat{\mathrm{X}}_{\phi}, \hat{\mathrm{X}}_{\phi+\pi / 2}\right]\right|^{2}=\frac{1}{4}\left\langle\hat{\mathrm{N}}_{1}+\hat{\mathrm{N}}_{2}+1\right\rangle$.

We will look at the effect of correlations between different modes on sum squeezing. For a generalized quadrature, $\hat{\mathrm{X}}_{\phi}=\frac{1}{2}\left(\mathrm{e}^{\mathrm{i} \phi} \hat{\mathrm{a}}+\mathrm{e}^{-\mathrm{i} \phi} \hat{\mathrm{a}}^{\dagger}\right)$, the variance can be expressed as

$$
\begin{aligned}
\Delta \hat{X}_{\phi}^{2}= & \frac{1}{4}+\frac{1}{4}\left[\mathrm{e}^{2 \mathrm{i} \phi}\left(\left\langle\hat{\mathrm{a}}^{\dagger}\right\rangle-\left\langle\hat{\mathrm{a}}^{\dagger}\right\rangle^{2}\right)+\mathrm{e}^{-2 \mathrm{i} \phi}\left(\left\langle\hat{\mathrm{a}}^{2}\right\rangle-\langle\hat{\mathrm{a}}\rangle^{2}\right)+\right. \\
& \left.2\left(\left\langle\hat{\mathrm{a}}^{\dagger} \hat{\mathrm{a}}\right\rangle-\langle\hat{\mathrm{a}}\rangle\left\langle\hat{\mathrm{a}}^{\dagger}\right\rangle\right)\right] .
\end{aligned}
$$


A state is squeezed if the bracketed term becomes negative, to attain the smallest value one must have $\arg \left(\left\langle\hat{a}^{2}\right\rangle-\langle\hat{a}\rangle^{2}\right)-2 \phi=\pi$, this gives

$\Delta \hat{\mathrm{X}}_{\phi}^{2}=\frac{1}{4}+\frac{1}{2}\left(\langle\hat{\mathrm{n}}\rangle-|\langle\hat{\mathrm{a}}\rangle|^{2}-\left|\left\langle\hat{\mathrm{a}}^{2}\right\rangle-\langle\hat{\mathrm{a}}\rangle^{2}\right|\right)$.

The squeezing criterion is then identical to the following inequality

$\langle\hat{n}\rangle-|\langle\hat{a}\rangle|^{2}<\left|\left\langle\hat{a}^{2}\right\rangle-\langle\hat{a}\rangle^{2}\right|$.

Analogously, it can be shown that for sum squeezing one must have

$\left\langle\hat{\mathrm{n}}_{\mathrm{a}} \hat{\mathrm{n}}_{\mathrm{b}}\right\rangle-|\langle\hat{\mathrm{a}} \hat{\mathrm{b}}\rangle|^{2}<\left|\left\langle\hat{\mathrm{a}}^{2} \hat{\mathrm{b}}^{2}\right\rangle-\langle\hat{a} \hat{b}\rangle^{2}\right|$.

Now, we will look at the effect of correlations between the constituent modes on sum squeezing. If the modes are uncorrelated, the sum squeezing condition (27) reduces to

$\left\langle\hat{\mathrm{n}}_{\mathrm{a}}\right\rangle\left\langle\hat{\mathrm{n}}_{\mathrm{b}}\right\rangle-|\langle\hat{\mathrm{a}}\rangle\langle\hat{\mathrm{b}}\rangle|^{2}<\left|\left\langle\hat{\mathrm{a}}^{2}\right\rangle\left\langle\hat{\mathrm{b}}^{2}\right\rangle-\langle\hat{\mathrm{a}}\rangle^{2}\langle\hat{\mathrm{b}}\rangle^{2}\right|$.

For the case where none of the individual modes is squeezed, we have from (26),

$$
\begin{aligned}
& \left\langle\hat{n}_{\mathrm{a}}\right\rangle-|\langle\hat{\mathrm{a}}\rangle|^{2} \geq\left|\left\langle\hat{\mathrm{a}}^{2}\right\rangle-\langle\hat{\mathrm{a}}\rangle^{2}\right|, \\
& \left\langle\hat{\mathrm{n}}_{\mathrm{b}}\right\rangle-|\langle\hat{\mathrm{b}}\rangle|^{2} \geq\left|\left\langle\hat{\mathrm{b}}^{2}\right\rangle-\langle\hat{\mathrm{b}}\rangle^{2}\right| .
\end{aligned}
$$

Now to look at the sum squeezing between the two modes

$$
\left|\left\langle\hat{a}^{2}\right\rangle\left\langle\hat{b}^{2}\right\rangle-\langle\hat{a}\rangle^{2}\langle\hat{b}\rangle^{2}\right| \leq\left\langle\hat{n}_{a}\right\rangle\left\langle\hat{n}_{b}\right\rangle-|\langle\hat{a}\rangle\langle\hat{b}\rangle|^{2},
$$

which shows on comparison with (27) that the state is not sum-squeezed.

\section{RESUlts AND Discussion}

We find that the quantum vortex state under investigation exhibit strong nonclassicalities as defined in Sections 3(A) to 3(D). For example, for the squeezing condition defined in section 3(A), we plot the $\mathrm{X}_{\mathrm{ab}}=\left\langle\left(\Delta \hat{\mathrm{X}}_{1}^{\mathrm{ab}}\right)^{2}\right\rangle-1 / 4$ as function of $\theta$ for different values of squeezing parameter $r$. We can see from the plot (Fig. 1) that for the smaller values of $r(=$ $0.1,0.2)$, there is no modal squeezing for any value of $\theta$.

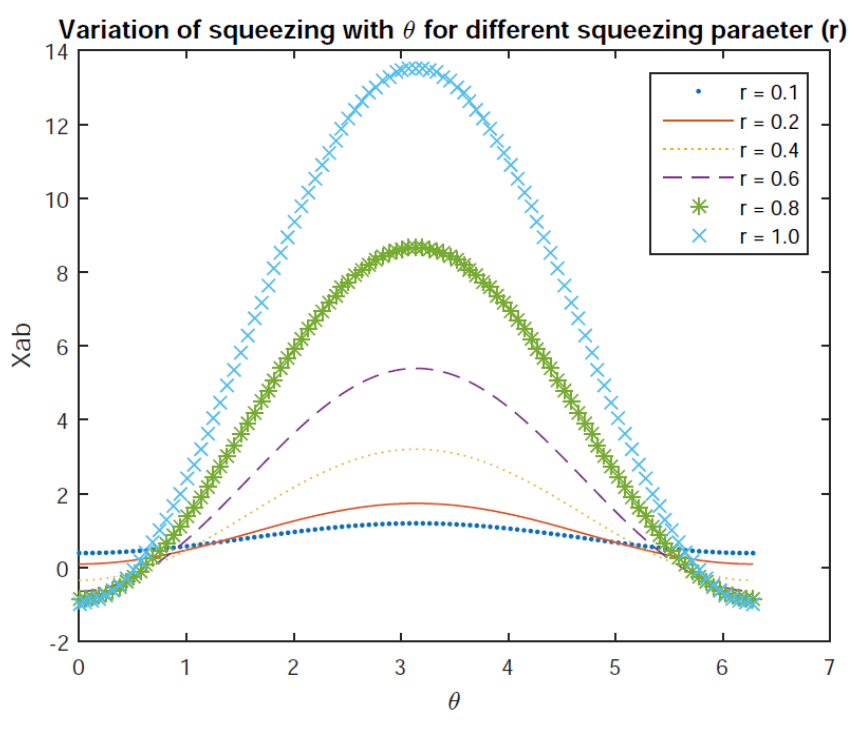

Fig. 1. Effect of squeezing on modal squeezing

As we increase the values of $r$, we find stronger modal squeezing but not for all values of $\theta$. As we increase $\theta$, the modal squeezing diminishes, and again modal squeezing appears for specific values of $\theta$. Similarly, we see other nonclassical properties, discussed in Section 4, are exhibited by the quantum vortex state dependent on the squeezing parameter $r$ and the angle $\theta$. This study shows the importance of two-mode quantum vortex state for possible application in quantum information processing where the nonclassicality is exploited as a resource.

\section{CONCLUSION AND FUTURE SCOPE}

We considered a non-Gaussian quantum two-mode vortex state and we studied its nonclassical properties in terms of squeezing, antibunching, photon number correlation and distribution of photon number. In addition, we studied higherorder nonclassical properties, viz., sum squeezing. We find that this non-Gaussian state shows strong nonclassical effects. It is our strong hope that the state under investigation here will be investigated under realistic conditions in near future.

\section{ACKNOWLEDGMENT}

Authors acknowledge UGC, New Delhi for financial support under Major Research Project (F. No. 41-950/2012(SR), Dated 26 July, 2012).

\section{REFERENCES}

[1] C. C. Gerry, P. L. Knight, Introductory quantum optics (Cambridge University Press; 2005)

[2] G. S. Agarwal, "Engineering non-Gaussian entangled states with vortices by photon subtraction”, New J. Phys., 13, 073008(2011). 
[3] A. Ourjoumtsev, R. Tualle-Brouri, J. Laurat and P. Grangier, "Generating optical Schrodinger kittens for quantum information processing”, Science 312, 83 (2006); T. Gerrits, S. Glancy, T. S. Clement, B. Calkins, A. E. Lita, A. J. Miller, A. L. Migdall, S. W. Nam, R. P. Mirin and E. Knill, "Generation of optical coherent-state superpositions by number-resolved photon subtraction from the squeezed vacuum", Phys. Rev. A 82, 031802 (2010); A. Biswas and G. S. Agarwal, "Nonclassicality and decoherence of photon-subtracted squeezed states", Phys. Rev. A 75, 032104 (2007).

[4] C. T. Lee, "Many-photon antibunching in generalized pair coherent states", Phys. Rev. A , 41, 1569 (1990).

[5] M. Hillery, "Sum and difference squeezing of the electromagnetic field”, Phys. Rev. A 40, 6 (1989).

\section{AUTHORS PROFILE}

Mr. Vikram Singh joined V. S. Mehta College of Science (Bhavan's Mehta Mahavidyalaya), Bharwari, District-Kaushambi, U. P. as a UGC project fellow in 2013 just after he pursued his M. Sc. Degree. He is currently pursuing his $\mathrm{Ph}$. D. degree at Department, Nehru Gram Bharati (Deemed to be University), Kotwa-Jamunipur Dubawal, Allahabad, U. P., India under the supervision of Dr. Devendra Kumar Mishra.

Dr. Devendra Kumar Mishra pursed Bachelor of Science, Master of Science (Physics), and D. Phil. from University of Allahabad, India. He is currently currently working as Associate Professor in Department of Physics, Institute of Science, Banaras Hindu University, Varanasi, India since 2017. Previously, he worked as

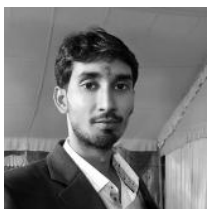
Assistant Professor and Associate Professor at Physics Department, V. S. Mehta College of Science (Bhavan's Mehta Mahavidyalaya), Bharwari, District-Kaushambi, U. P., India since 2003. He is a recepient of international Raman fellowship awarded by UGC, India. He has several publications in international refereed journals having high impact factors in the area of quantum optics and quantum information. 